\title{
Circular Dichroism of the "Random" Polypeptide Chain
}

\author{
M. LOIS TIFFANY and S. KRIMM, Biophysics Research Division, \\ Institute of Science and Technology and Harrison M. Randall \\ Laboratory of Physics, University of Michigan, Ann Arbor, \\ Michigan 48107
}

\section{Synopsis}

The circular dichroism (CD) spectrum of an unordered polypeptide chain does not correspond, as has been assumed heretofore, to that of a charged chain such as poly-Lglutamic acid or poly-I-lysine. The latter have been shown to have locally ordered structures with characteristic CD spectra. We have now obtained CD spectra of the unordered forms of the above synthetic polypeptides, as well as of two fibrous proteins (collagen and feather keratin) and a globular protein (myoglobin). These spectra are all similar to that of unordered polyproline, having a negative band in the vicinity of $200 \mathrm{~m} \mu$ and no additional bands at longer wavelengths. The lack of structural uniqueness of the unordered polypeptide chain is emphasized by these studies.

\section{INTRODUCTION}

The utility of circular dichroism (CD) in studying the conformation of polypeptides and proteins depends upon the possibility of correlating specific structures with characteristic CD spectra. Thus, for example, the CD spectrum of the $\alpha$-helix has been characterized ${ }^{1-3}$ in terms of the wavelengths and ellipticities of its three bands at about 190, 207, and $222 \mathrm{~m} \mu$. Similar studies have been done in order to define the CD spectrum of the so-called "random" form of the polypeptide chain. ${ }^{2-7}$ These have all been based on the assumption that a polypeptide chain with charged side chain groups is a satisfactory model for a random coil or unordered structure. It has been pointed out, however, both from experimental ${ }^{8}$ as well as theoretical ${ }^{8,9}$ arguments, that such chains have a local extended-helix structure. Therefore, from the CD criterion their spectra cannot be expected to be representative of that of an unordered chain.

The form of the CD spectrum believed to be associated with an unordered polypeptide chain was first described as that corresponding to denatured collagen. ${ }^{10}$ A qualitatively similar curve has since been found to characterize an unordered conformation of poly-L-proline (PP). ${ }^{11}$ In this paper we report the existence of analogous CD spectra for poly(L-glutamic acid) (PGA) and poly-L-lysine (PL), as well as for various solubilized fibrous proteins. The evidence from these as well as other cases suggests that the 
form of the CD spectrum of an unordered polypeptide chain can now be considered as established. The relevance of this to the interpretation of the CD spectra of globular proteins will also be considered.

\section{Inadequacy of Polyelectrolytes as Models for an Unordered Polypeptide Chain}

It will be useful to first review the arguments which indicate that polypeptide chains with charged side chain groups are not satisfactory models, from the point of view of $\mathrm{CD}$, for an unordered chain.

Although it has long been realized that, as a result of electrostatic repulsions, charged polypeptide chains have a generally extended chain structure, it has been assumed that this conformation can be validly described as a random coil. Such a conclusion implies that there is no long range regularity in charged chains, but it of course does not preclude the possibility that a locally ordered structure may exist. In fact, it was first pointed out by Tiffany and Krimm ${ }^{8}$ that the CD spectra of charged PGA and PL in the absence of additional salt strongly suggest the presence of an extended-helix structure with parameters close to those of the threefold left-handed helix of PP II. They also noted that simple considerations showed that such a structure would be expected for PGA on the basis of minimization of electrostatic interactions along the chain.* Thus, the chain was envisioned to have bends in it, the portions between bends being in the extended-helix conformation and containing a number of residues sufficient to give a characteristic CD spectrum.

The parameters of the extended-helix structure have been determined in greater detail by a calculation of the minimum energy conformation of an actual charged polypeptide chain. ${ }^{9}$ This calculation shows that the favored structure is a left-handed helix having between about 2.6 and 2.8 residues per turn (depending on the number of peptide units in the segment), and that the helix symmetry is independent of side-chain length for groups of the size of glutamic acid or longer. Both of these predictions are in accord with the experimental evidence. ${ }^{8}$

It is therefore to be expected that axially periodic side-chain charges will exert a rigidifying and regularizing effect on the polypeptide chain backbone, giving rise to locally ordered extended-helix structures with characteristic CD spectra. For left-handed helices of $\mathrm{L}$-amino acids with close to three residues per turn, these spectra are characterized by a small positive peak near $220 \mathrm{~m} \mu$ and a large negative peak near $200 \mathrm{~m} \mu,{ }^{8}$ spectra which are qualitatively similar to that for the left-handed helix of PP II. The presence in some instances of an additional weak negative band near $235 \mathrm{~m} \mu$ is believed to be associated with an intermediate conformational state. $^{8}$ It is interesting to note that the above assignment of the CD spectrum of the left-handed extended-helix conformation is supported by

* For computational purposes a similar structural assumption was made by Zimm and Rice $^{12}$ in their treatment of the helix-coil transition in PGA, although no reason was given for doing this. 
the general features of the $\mathrm{CD}$ spectrum of poly- $N$-methyl-L-alanine. ${ }^{13}$ This polypeptide, which conformational energy calculations show to be an approximately three-fold right-handed helix, ${ }^{14,15}$ has a CD spectrum exhibiting a negative band at $223 \mathrm{~m} \mu$ and a positive band at $192 \mathrm{~m} \mu$. This curve is, as expected, of opposite sign compared to that for a left-handed helix.

The recognition that side-chain charges impose a particular regularity on the main-chain conformation can help to explain the unusual CD spectrum of phosvitin. ${ }^{3}$ This protein, about $78 \%$ of whose amino acid residues can be charged (about $60 \%$ being negatively charged at high $\mathrm{pH}$ ), has a CD spectrum similar to that of charged PGA. The spectrum was found to be difficult to interpret, and its obvious similarity to that of collagen (which basically has a PP II chain conformation) was rejected because of the very small amount of proline present $(1.5 \%)$. We can now more confidently interpret this spectrum in terms of the presence of a PP II type of conformation since we now know that this is precisely the kind of structure which is stabilized by side-chain electrostatic interactions.

\section{Synthetic Polypeptides in the Unordered Conformation}

The above analysis of the CD spectra of charged polypeptide chains show's that the presence in the spectrum of a weak positive band near 220 $\mathrm{m} \mu$, together with the large negative band near $200 \mathrm{~m} \mu$, is indicative of the occurrence of a specific local structure (the extended helix) rather than of a true random conformation. The disappearance of this positive band, first noted for denatured collagen, ${ }^{10}$ is to be associated with a transformation to an unordered structure. Such a spectrum is now also found for synthetic polypeptides, and in this section we discuss the results for such systems.

As noted earlier, polyproline has been found ${ }^{11}$ to be capable of giving a CD spectrum indicating that it can be in an unordered conformation. This spectrum, which consists essentially of one negative band at $205 \mathrm{~m} \mu$, with $\Delta \epsilon=-6$ to -7 , is obtained from PP I or PP II when this polypeptide is placed in 4-6M $\mathrm{CaCl}_{2}$. (The latter solvent was quite unexpectedly found to be sufficiently transparent in the far ultraviolet to permit CD measurements down to $200-205 \mathrm{~m} \mu$.) This curve is qualitatively similar to that of denatured collagen (see below), and appears to be associated in

$\mathrm{PP}$ with an effective randomization about the $\mathrm{C}^{\alpha}-\mathrm{C}$ bond. The loss of correlation of structure along the chain is believed to be the basis for the disappearance of the characteristic PP I or PP II CD spectrum.

In the case of PGA, we have shown ${ }^{8}$ that a transformation away from the CD spectrum of the extended-helix structure occurs by lowering the $\mathrm{pH}$ from $\mathrm{pH} 7$ or by adding methanol to a $\mathrm{pH} 7$ solution of PGA. (Since for both agents the transformation to an $\alpha$-helix involves an isodichroic point in the $\mathrm{CD}$ spectrum, ${ }^{8}$ the intermediate structural states are pre- 


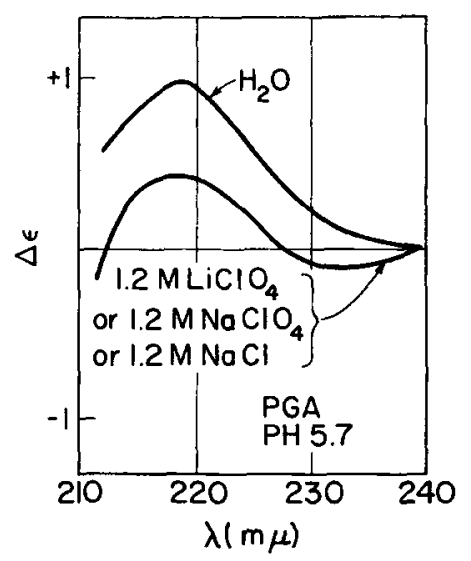

Fig. 1. CD spectra of poly(t-glutamic acid) (PGA) in $\mathrm{H}_{2} \mathrm{O}$ at pH $5.7(c=0.69 \mathrm{~g} / \mathrm{l}$.)

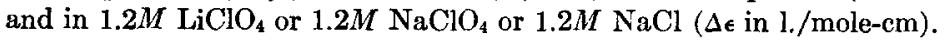

dominantly mixtures of $\alpha$-helix and extended-helix conformations.) A similar effect is produced by the addition of salt, as is shown in Figure 1. $\left(\mathrm{LiClO}_{4}\right.$ must be used since addition of $\mathrm{CaCl}_{2}$ results in precipitation of the PGA.) It will be seen that the departure from extended-helix structure indicated by the weakening of the positive band at $218 \mathrm{~m} \mu$, is accompanied by the apparent appearance of a negative band near $235 \mathrm{~m} \mu$. As we observed earlier, ${ }^{8}$ we do not think that this represents a separate CD band, but rather is characteristic of an intermediate structural state of the system.

We have now found it possible to obtain PGA in an essentially unordered state. This is achieved by heating a solution of the polypeptide in $4.5 \mathrm{M}$ $\mathrm{LiClO}_{4}$ to $50^{\circ} \mathrm{C}$ for $\frac{1}{4} \mathrm{hr}$ and then cooling it. The CD spectrum in this state is shown in Figure 2, where for comparison the CD spectrum of PGA

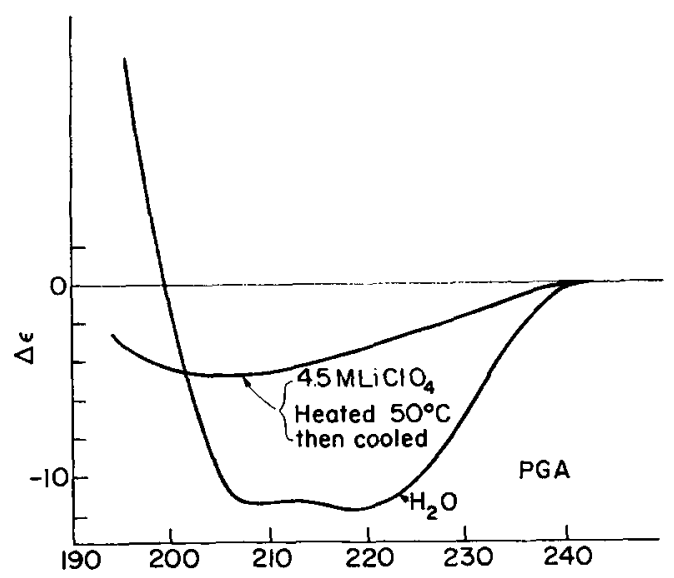

Fig. 2. $\mathrm{CD}$ spectra of PGA in $4.5 \mathrm{M} \mathrm{LiClO}$ and in $\mathrm{H}_{2} \mathrm{O}$ at $\mathrm{pH}<5(c=1.71 \mathrm{~g} / \mathrm{l}$. $)$. 
in its $\alpha$-helical state (in water at pH lower than about 5) is shown. It will be seen that the former curve is qualitatively similar to that of the unordered PP, its minimum oceurring at about $205 \mathrm{~m} \mu$ with $\Delta \epsilon \cong-5$. There is no positive band near $220 \mathrm{~m} \mu$, confirming our contention that the latter is not to be correlated with an unordered structure.

It is interesting to note that guanidine hydrochloride, which is a very effective denaturing agent for proteins, ${ }^{16}$ also has the effect of transforming the structure of charged PGA from the extended-helix conformation to that of a more disordered state. In Figure 3 are shown the CD spectra of PGA in the $220 \mathrm{~m} \mu$ region at $\mathrm{pH} 7$ in the absence of added salt and after making the solution $3 M$ in guanidine hydrochloride. It will be seen that in the latter case the peak near $220 \mathrm{~m} \mu$ is eliminated, the curve displaying the characteristics of an essentially disordered chain. This trend is much more poorly developed in $3 M \mathrm{IiCl}$, as can be seen from the curve for the latter in Figure 3, thus showing the greater efficacy of guanidine hydrochloride in disorganizing the polypeptide chain.

An unordered state spectrum has now also been obtained for PL. At high $\mathrm{pH}, \mathrm{PL}$ occurs in the $\alpha$-helix conformation. ${ }^{5}$ At low $\mathrm{pH}$, when the side chains are charged, it has been assumed ${ }^{3,5,7,17}$ that the chain conformation is a random one. Under these conditions the spectrum is similar to that of PGA at high $\mathrm{pH}$, and therefore we believe that this curve indicates that $\mathrm{PL}$ has an extended-helix structure in this case. ${ }^{8}$ This is confirmed by theoretical studies. The CD spectrum which we now associate with the unordered polypeptide chain can be obtained with PL in $4 M \mathrm{CaCl}_{2}$. This is shown in Figure 4, where for purposes of comparison the $\alpha$-helix spectrum ( $\mathrm{pH} \mathrm{11)} \mathrm{and} \mathrm{the} \mathrm{extended-helix} \mathrm{spectrum} \mathrm{(} \mathrm{pH}$ 6) are

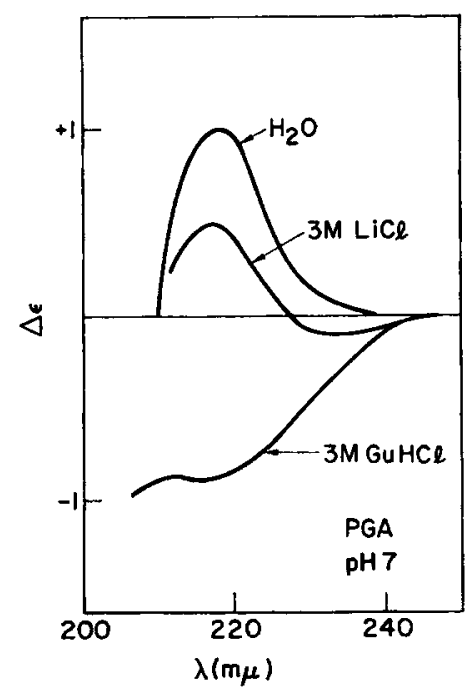

Fig. 3. $\mathrm{CD}$ spectra of $\mathrm{PGA}$ in $\mathrm{H}_{2} \mathrm{O}, 3 M \mathrm{LiCl}$, and $3 M$ guanidine hydrochloride $(c=1.71 \mathrm{~g} / 1$.$) .$ 


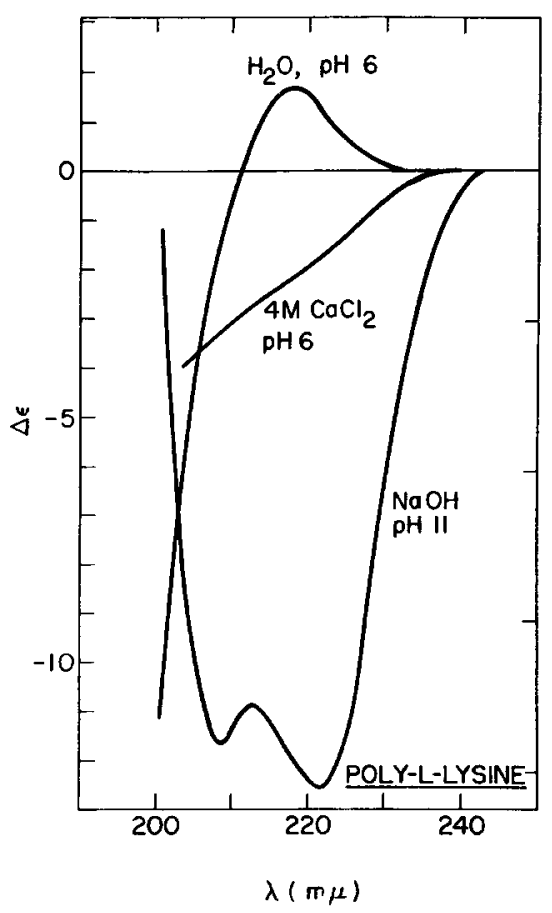

Fig. 4. $\mathrm{CD}$ spectra of poly-t-lysine in $\mathrm{H}_{2} \mathrm{O}(\mathrm{pH} 6), 4 M \mathrm{CaCl}_{2}\left(\mathrm{pH}\right.$ 6), and $\mathrm{H}_{2} \mathrm{O}$ (pH 11 with $\mathrm{NaOH})(c=0.666 \mathrm{~g} / \mathrm{l}$. $)$.

also shown. It will be seen that the positive band near $220 \mathrm{~m} \mu$ has disappeared, and a negative band near $200 \mathrm{~m} \mu$, with $\Delta \epsilon \cong-4$, is indicated. Thus, PL also appears to be capable of assuming a conformation in salt solution which can be characterized as essentially unordered.

There is evidence in the literature for similar CD spectra for other synthetic polypeptides, although the above interpretation was not clearly recognized. Thus, poly-L-serine in $8 M \mathrm{LiCl}$ shows only a predominant negative band near $200 \mathrm{~m} \mu$, with $\Delta \epsilon \cong-2.5 .^{18}$ On the basis of the presumed destabilization of polypeptides by high concentrations of lithium salts, ${ }^{19,20}$ it was conjectured ${ }^{18}$ that the poly-L-serine was in a disordered state, even though its spectrum did not resemble that of high pH PGA. Our studies verify this supposition, and resolve the dilemma by showing that the PGA referred to is in fact not random but has a locally regular extended-helix structure.

It is pertinent to note at this point that the $\mathrm{CD}$ spectrum which we associate with an unordered conformation of the polypeptide chain, while of a characteristic qualitative form, is not quantitatively identical for all synthetic polypeptides. While the position of the single negative band is near $200 \mathrm{~m} \mu$, in some cases (PP, PGA) it is at higher and in other cases (PL, poly-L-serine) it is at lower wavelengths. The value of $\Delta \epsilon$ also varies significantly, from -6 to -7 in the case of $P P$ to -2.5 in the case of 
poly-L-serine. The values for PP may reflect the imide structure of this polypeptide, but the differences between PGA and PL on the one hand and poly-L-serine on the other could well be real. Such differences may be attributable to the fact that there is no single well-defined unordered structure, but rather a range of structures depending on the values of $\phi$ and $\psi$ along the chain. Different values of $\phi$ and $\psi$ in the peptide group as well as different degrees of correlation along the chain could give rise to CD spectra which vary somewhat in the position and intensity of the main bands.

\section{Fibrous Proteins in the Unordered Conformation}

The correlation of the CD spectrum of heat-denatured collagen with that of an unordered polypeptide chain structure ${ }^{10}$ has already been noted. The CD spectrum of such a sample is shown in Figure 5, as well as that of the native sample prior to heat denaturation. In the same figure is the CD spectrum of a native sample treated with $3.6 M \mathrm{LiClO}_{4}$. It will be seen that the spectrum of the heat-denatured sample is analogous to that which we have associated with the unordered polypeptide chain. In fact, the independent evidence for separation of the three strands of the collagen structure upon such heat treatment and their randomization ${ }^{12}$ provides perhaps the strongest basis for establishing the characteristic CD spectrum

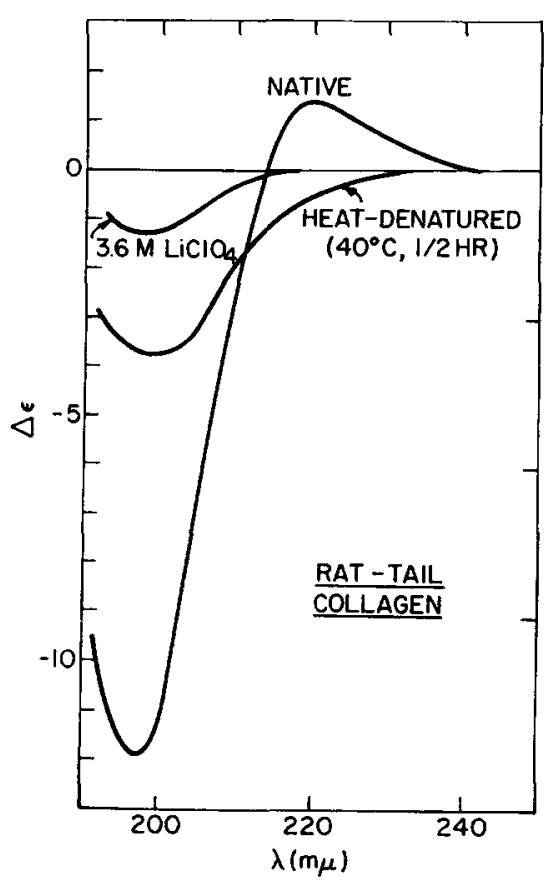

Fig. 5. CD spectra of rat-tail collagen in the native state, heat denatured $\left(40^{\circ} \mathrm{C}\right.$ for $\left.\frac{1}{2} \mathrm{hr}\right)$, and in $3.6 \mathrm{M} \mathrm{LeClO}_{4}(c=0.345 \mathrm{~g} / \mathrm{l}$.). 
of the unordered chain. The extent of denaturation depends on the time of heating, as measured by the intensity of the band at $220 \mathrm{~m} \mu$ (see Fig. 6). For both the heat-denatured and $\mathrm{LiClO}_{4}$-denatured samples, the minimum is found at $195-198 \mathrm{~m} \mu$. However, in the former case, $\Delta \epsilon \cong-4$ whereas in the latter, $\Delta \epsilon \cong-1$. This difference could be associated with the effect of specifically bound salt on the transitions of the peptide group, or it could be due to conformational differences between the two kinds of chains. In view of the similar low value obtained for solubilized feather keratin in the absence of salt (see below), we are more inclined to attribute this to the effect of the salt in altering the chain conformation. This again points up the fact that the "random" conformation represents no single structure but is to be thought of as encompassing a range of structures.

Other reagents will also denature collagen. For example, $5 M$ urea was found to eliminate the $220 \mathrm{~m} \mu$ peak in less than $1 \mathrm{~min}$. However, if $2 M$ urea is used, only partial reduction of this peak is observed, the intensity diminishing to about one-third of the native value after $2 \mathrm{hr}$ (no further change occurs with time). Denaturation also occurs in $3 M \mathrm{CaCl}_{2}$ solution, as indicated by the disappearance of the $220 \mathrm{~m} \mu$ peak.

From optical rotatory dispersion measurements, it was concluded ${ }^{22}$ that feather keratin which had been solubilized in urea plus bisulfite was in an essentially random conformation. We believe that this is now confirmed by CD measurements. The CD spectrum of such a sample is shown in Figure 7 in an aqueous solution (the urea and bisulfite having been removed by dialysis). It will be seen that this curve, with the indication of

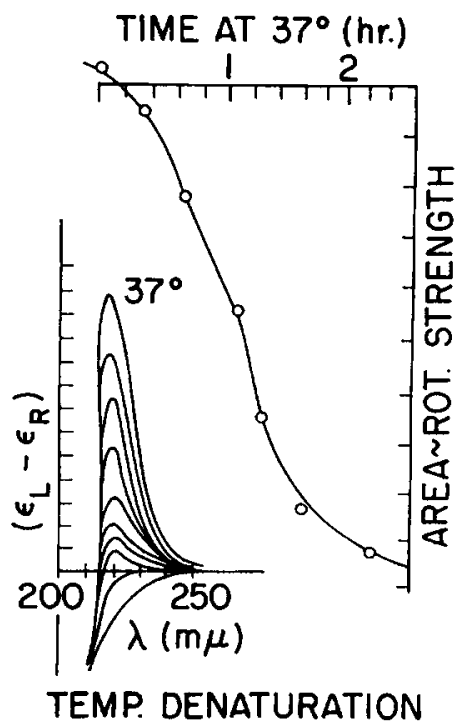

Fig. 6. CD spectra of heat-denatured collagen as a function of time of denaturation at $37^{\circ} \mathrm{C}$. The area of the peak at $220 \mathrm{~m} \mu$ is plotted as a fraction of its value in the native state as a function of time. 


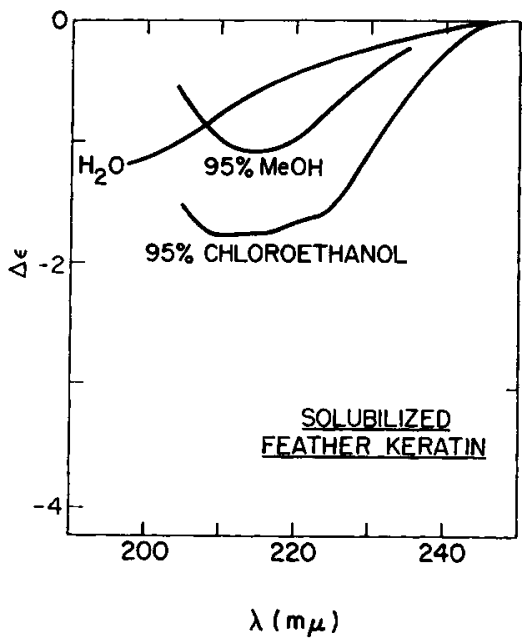

Fig. 7. CD spectra of solubilized feather keratin in $\mathrm{H}_{2} \mathrm{O}$, in $95 \%$ methanol, and in $95 \%$ chloroethanol. $\quad(c=1.95 \mathrm{~g} / \mathrm{l}$.$) .$

a negative peak just below $200 \mathrm{~m} \mu$, is analogous to those of the previously discussed unordered structures. The value of $\Delta \epsilon \cong-1$ is close to that of collagen denatured with $\mathrm{LiClO}_{4}$ (see Fig. 5). That the structure of solubilized feather keratin can be strongly influenced by the solvent is shown in the other two curves in Figure 7 . In 95\% methanol the spectrum exhibits a negative band at about $215 \mathrm{~m} \mu$, reminiscent of that characteristic of a $\beta$ structure ${ }^{3}$, whereas in $95 \%$ chloroethanol features characteristic of the $\alpha$-helix appear as well.

The CD spectra of fibrous proteins which are known from other techniques to be in a highly unordered conformation are thus seen to be similar to those of unordered synthetic polypeptides.

\section{Circular Dichroism Spectra of Globular Proteins}

The characterization of the CD spectrum of the unordered polypeptide chain, which we have seen to be possible from a study of synthetic polypeptides and fibrous proteins, should permit a more cogent qualitative analysis to be made of the conformational structures of globular proteins in their native and nonnative states. In this section we illustrate this point by results on several globular proteins.

Myoglobin is well known to be predominantly $\alpha$-helical (about $75 \%$ ), and its $\mathrm{CD}$ spectrum in water confirms this (Fig. 8). When placed in $4.8 M \mathrm{CaCl}_{2}$, however, the spectrum undergoes a dramatic change (see Fig. 8) to one characteristic of an unordered structure. It is clear in this simple case that the salt has brought about an essentially complete conversion of the ordered $\alpha$-helical structure to a form similar to those of the unordered conformations of other polypeptides. This state is substantially reversed by dilution of the solution to one-fifth of the original $\mathrm{CaCl}_{2}$ con- 


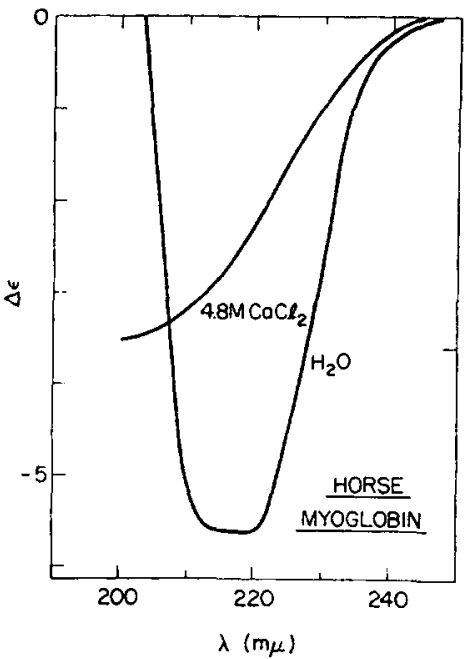

Fig. 8. CD spectra of horse myoglobin in $\mathrm{H}_{2} \mathrm{O}$ and in $4.8 M \mathrm{CaCl}_{2}(c=1.08 \mathrm{~g} / \mathrm{l}$.$) .$

centration: the CD spectrum returns immediately to its original form, with an intensity about $80 \%$ of the original (the failure to return $100 \%$ seems to be due in part to precipitation of some myoglobin).

Ribonuclease in its native state gives a CD spectrum which is consistent with the presence of some $\alpha$-helical component plus a dominant unordered component (Fig. 9). This is in agreement with the known x-ray structure of this protein. Some reagents, such as methanol and chloroethanol, appear to enhance the $\alpha$-helical content over that of the native protein (in

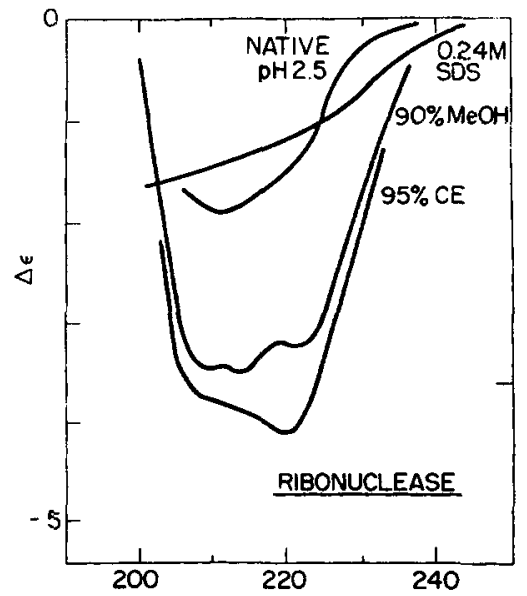

$\lambda(m \mu)$

Fig. 9. CD spectra of ribonuclease in the native state (at pH 2.5), in $0.24 M$ sodium dodecyl aulfate, in $90 \%$ methanol, and in $95 \%$ chloroethanol $(c=0.312 \mathrm{~g} / \mathrm{l}$.). 


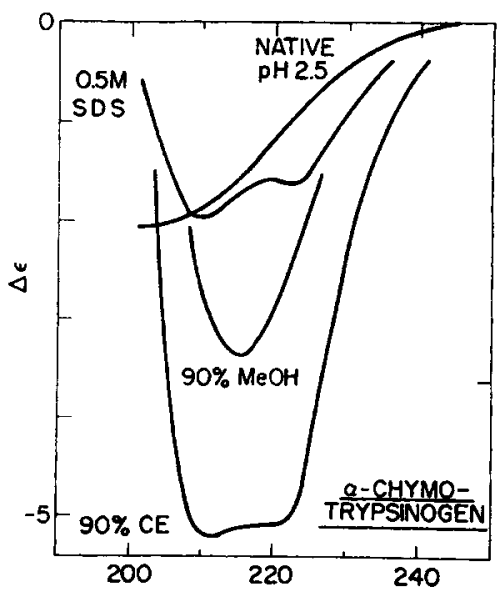

$\lambda\left(m_{\mu}\right)$

Fig. 10. CD spectra of $\alpha$-chymotrypsinogen in the native state (at pH 2.5 ), in $0.5 M$ sodium dodecyl sulfate, in $90 \%$ methanol, and in $90 \%$ chloroethanol $(c=0.512 \mathrm{~g} / \mathrm{l}$.).

the former case possibly with a concomitant appearance of some $\beta$ structure). This may, of course, occur through an intermediate unordered structure. Other reagents such as sodium dodecyl sulfate reduce the helical content and cause the CD spectrum to approach more closely that of an unordered chain (see Fig. 9).

For $\alpha$-chymotrypsinogen, on the other hand, although the native protein has a very specific structure, the CD spectrum is similar to that of an essentially unordered chain. Addition of sodium dodecyl sulfate acts to enhance the $\alpha$-helical content (Fig. 10). In this case methanol appears to produce predominantly a $\beta$ structure, whereas chloroethanol leads to the development of a strong $\alpha$-helix spectrum.

The above comments have been of necessity qualitative since, as we have seen, the CD spectrum of the unordered chain is not an immutable curve but depends on the "degree of disorder" of the particular polypeptide. It is probably also true that the CD spectrum of the $\alpha$-helix is dependent on local order and rigidity. Quantitative estimates of component structures in globular proteins from analyses of CD spectra must therefore be approached with considerable caution at the present time.

\section{DISCUSSION}

The possibility of associating a characteristic CD spectrum with an unordered conformation of a polypeptide chain is seen from the above results to be feasible from a qualitative but not from a quantitative standpoint. We consider below some aspects of this situation.

The experimental evidence suggests that the CD spectrum of an unordered polypeptide chain is characterized by a single broad negative band 
centered near $200 \mathrm{~m} \mu$. A curve of this kind is obtained from a variety of different starting conformations: the two helical structures of polyproline, ${ }^{11}$ the triple-stranded helix of collagen, the extended helix of charged polypeptides, ${ }^{8}$ and the predominantly $\alpha$-helical structure of myoglobin. Furthermore, it can be produced under a variety of conditions: in the presence of various salts (PP, PGA, PL), in the absence of salts (soluble feather keratin), and as a result of denaturation by heat (collagen). We believe that this indicates that such a curve is to be associated with a particular new kind of chain conformation rather than being due to the effect of some interaction on the original structure. The common feature of these treatments is that they tend to destroy the native structure, and we are therefore led to associate this spectrum with an unordered polypeptide chain.

It is perhaps worth noting that the term unordered is to be preferred to "random coil" in designating such structures. This is because although the overall structure of a chain may well be validly described as a random coil, this implies no specification on the presence or absence of local order. That is, a random coil is consistent with the existence of statistical elements of varying size. These in turn may imply different degrees of local order, and since the CD spectrum is sensitive to the latter characteristic of the chain, we may expect the possibility that structures which are random from the overall point of view nevertheless have different CD spectra. Thus, we believe that while the overall chain structure of PGA at high pH may be considered to be random, at the local level the peptide group orientations are correlated over long enough sequences to give a characteristic extended-helix CD spectrum. ${ }^{8}$ The term "unordered" is therefore meant to signify an absence of correlation in $\phi, \psi$ for neighboring peptide groups, thus destroying any specific features of the CD spectrum which arise from such correlation. It should be noted that intermediate degrees of correlation are possible; this may explain structural features seen in the $210-230 \mathrm{~m} \mu$ region of the CD spectrum in some cases. ${ }^{8,18}$

Although the qualitative features of all of the curves obtained for polypeptides in the unordered state are the same, this is not true of the quantitative characteristics. Thus, $\Delta \epsilon_{200}$ is found to vary significantly between different systems. It is not possible at present to give a detailed accounting of the origin of this variability, but the influence of certain factors may be surmised. Thus, if the average $\phi, \psi$ were to depend on side-chain composition, this could give rise to characteristically different $\Delta \epsilon$ values for different polypeptide chains. Changes in $\Delta \epsilon$ for a given chain, such as in the case of collagen denatured by heat or by salts, may reflect the changes in average $\phi, \psi$ originating from some specific effect of the environment. This again emphasizes the fact that the unordered chain represents no single structure but encompasses a range of structural possibilities.

This variable feature of the CD spectrum of the unordered polypeptide chain suggests that a simple attempt to resolve a curve for an arbitrary protein structure into contributions from component conformations may not be feasible. That is, no standard curve for a " $100 \%$ unordered struc- 
ture" can be considered to exist. Of course, a negative band near $200 \mathrm{~m} \mu$ can be assumed for the unordered portion of the structure, and its intensity determined by difference after fitting with standard curves for ordered chain eonformations. It is likely, however, that the fit, will not be unique in such a case on the basis of present knowledge. Better quantitative resolution will require spectra at shorter wavelengths in order to take into account bands which are present in this region.

This research was supported by U.S. Public Health Service grant AM 02830.

\section{References}

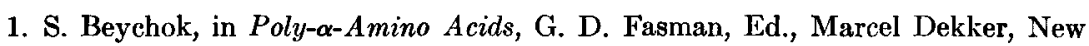
York, 1967, p. 293.

2. J. T. Yang, in Conformation of Biopolymers, G. N. Ramachandran, Ed., Academic Press, New York-London, 1967, p. 157.

3. S. N. Timasheff, H. Susi, R. Townend, L. Stevens, M. J. Gorbunoff, and T. F. Kumosinski, in Conformation of Biopolymers, G. N. Ramachandran, Ed., Academic Press, New York-London, 1967, p. 173.

4. M. Legrand and IR. Viennet, C. R. Acad. Sci. (Paris), 259, 4277 (1964).

5. G. Holzwarth and P. Doty, J. Amer. Chem. Soc., 87, 218 (1965).

6. L. Velluz and M. Legrand, Angrw. Chem. Intern. Ed. Eng. 4, 838 (1965).

7. J. P. Carver, E. Shechter, and E. R. Blout, J. Amer. Chem. Soc., 88, 2550 (1966).

8. M. L. Tiffany and S. Krimm, Biopolymers, 6, 1379 (1968).

9. S. Krimm and J. E. Mark, Proc. Nat. Acad. Sci., U.S., 60, 1122 (1968).

10. M. I. Tiffany and S. Krimm, Biophys. Soc. Abstr., TE 5 (1967).

11. M. L. Tiffany and S. Krimm, Biopolymers, 6, 1767 (1968).

12. B. H. Zimm and S. A. Rice, Nol. Phys., 3, 391 (1960).

13. M. Goodman and M. Fried, J. Amer. Chem. Soc., 89, 1264 (1967).

14. J. E. Mark and M. Goodman, J. Amer. Chem. Soc., 89, 1267 (1967).

15. J. E. Mark and M. Goodman, Biopolymers, 5, 809 (1967).

16. C. Tanford, K. Kawahara, S. Iapanje, T. M. Hooker, M. H. Zarlengo, A. Salahuddin, K. C. Aune, and T. Takaji, J. Amer. Chem. Soc., 89, 5023 (1967).

17. R. Townend, T. F. Kumosinsli, and S. N. Timasheff, Biochem. Biophys. Res. Comm., 23, 163 (1966).

18. F. Quadrifoglio and D. W. Urry, J. Amer. Chem. Soc., 90, 2760 (1968).

19. G. D. Fasman, C. Lindblow, and Li. Bodenheimer, Biochemistry, 3, 155 (1966).

20. G. Barone, V. Crescenzi, and F. Quadrifoglio, Biopolymers, 4, 529 (1966).

21. H. Boedtker and P. Doty, J. Amer. Chem. Soc., 78, 4267 (1956).

22. C. J. Westover, M. L. Tiffany, and S. Krimm, J. Mol. Biol., 4, 316 (1962).

Received February 14, 1969

Revised March 31, 1969 